\title{
A UNIFYING FRAMEWORK FOR THE CIRCLE CRITERION AND OTHER QUADRATIC STABILITY CRITERIA
}

\author{
R. N. Shorten ${ }^{1}$, O. Mason ${ }^{2}$, F. O'Cairbre ${ }^{3}$, P. Curran ${ }^{4}$ \\ ${ }^{1}$ Hamilton Institute, NUI Maynooth, Co. Kildare, Ireland, robert.shorten@may.ie, fax +35317086269 \\ ${ }^{2}$ Hamilton Institute, NUI Maynooth, Co. Kildare, Ireland, oliver.mason@may.ie \\ ${ }^{3}$ Dept. of Mathematics, NUI Maynooth, Co. Kildare, Ireland, foc@maths.may.ie \\ ${ }^{4}$ Dept. of Electrical Engineering, UCD, Dublin 4, Ireland, paul.curran@ucd.ie
}

Keywords: Switched Linear Systems, Lur'e Systems, Stability, Common quadratic Lyapunov functions, Kalman-Yakubovich-Popov Lemma

\begin{abstract}
We present a result on the existence of a common quadratic Lyapunov function for a pair of linear timeinvariant systems. We show that this result characterises, generalises, and provides new perspectives on several well-known stability results. In particular, new time-domain formulations of the Circle Criterion and Meyer's extension of the KYP lemma are presented.
\end{abstract}

\section{Introduction}

In this paper we consider the problem of determining necessary and sufficient conditions for the existence of a common quadratic Lyapunov function (CQLF) for a pair of stable linear time-invariant (LTI) systems. This problem arises in many areas of systems theory; in particular, in the study of non-linear Lur'e type systems and in the study of switched linear systems. While the general algebraic problem is extremely difficult, necessary and sufficient conditions for various system classes have been obtained by reposing the problem in the form of a linear matrix inequality, or (by means of the positive real lemma) as a frequencydomain optimization. We present a new approach to solving this problem. By formulating the CQLF existence problem in a set-theoretic context, and by making simplifying assumptions, we obtain a simple eigenvalue condition for the existence of a CQLF for a pair of LTI systems. We show that well known stability criteria are characterised by this result. In particular, we obtain a new time-domain formulation of the circle criterion.

\section{Mathematical preliminaries and nota- tion}

In this section we present preliminary results that are useful in deriving the main contribution of this paper. Throughout, the following notation is adopted: $\mathbb{R}$ and $\mathbb{C}$ denote the fields of real and complex numbers respectively; $\mathbb{R}^{n}$ denotes the $n$-dimensional real Euclidean space; $\mathbb{R}^{n \times n}$ denotes the space of $n \times n$ matrices with real entries; $x_{i}$ denotes the $i^{\text {th }}$ component of the vector $x$ in $\mathbb{R}^{n} ; a_{i j}$ denotes the entry in the $(i, j)$ position of the matrix $A$ in $\mathbb{R}^{n \times n}$.

The main results of this paper are based upon Theorem 2.1. The concepts of weak quadratic Lyapunov functions, strong quadratic Lyapunov functions, and matrix pencils, are central to the statement of this theorem. Where appropriate, proofs of individual theorems and lemmas are given in the appendix.

(i) Strong and weak CQLFs : Consider the set of LTI systems

$$
\Sigma_{A_{i}}: \dot{x}=A_{i} x, i \in\{1,2, \ldots M\} .
$$

where $M$ is finite and the $A_{i}, i \in\{1,2, \ldots M\}$, are constant Hurwitz matrices in $\mathbb{R}^{n \times n}$ (i.e. the eigenvalues of $A_{i}$ lie in the open left half of the complex plane and hence the $\Sigma_{A_{i}}$ are stable LTI systems). Let the matrix $P=P^{T}>0, \quad P \in$ $\mathbb{R}^{n \times n}$, be a simultaneous solution to the Lyapunov equations

$$
A_{i}^{T} P+P A_{i}=-Q_{i}, i \in\{1,2, \ldots M\} .(2)
$$

Then, $V(x)=x^{T} P x$ is a strong quadratic Lyapunov function for the LTI system $\Sigma_{A_{i}}$ if $Q_{i}>$ 0 , and is said to be a strong CQLF for the set of LTI systems $\Sigma_{A_{i}}, i \overline{\in\{1, \ldots, M\}}$, if $Q_{i}>0$ for all $i$. Similarly, $V(x)$ is a weak quadratic Lyapunov function for the LTI system $\Sigma_{A_{i}}$ if $Q_{i} \geq 0$, and is said to be a weak CQLF for the set of LTI systems $\Sigma_{A_{i}}, i \in \overline{\{1, \ldots, M\}}$, if $Q_{i} \geq 0$ for all $i$. 
(ii) The matrix pencil $\sigma_{\gamma[0, \infty)}\left[A_{1}, A_{2}\right]$ : The matrix pencil $\sigma_{\gamma[0, \infty)}\left[A_{1}, A_{2}\right]$, for $A_{1}, A_{2} \in$ $\mathbb{R}^{n \times n}$, is the parameterised family of matrices $\sigma_{\gamma[0, \infty)}\left[A_{1}, A_{2}\right]=A_{1}+\gamma A_{2}, \quad \gamma \in$ $[0, \infty)$. We say that the pencil is non-singular if $\sigma_{\gamma[0, \infty)}\left[A_{1}, A_{2}\right]$ is non-singular for all $\gamma \geq 0$. Otherwise the pencil is said to be singular. Further, a pencil is said to be Hurwitz if its eigenvalues are in the open left half of the complex plane for all $\gamma \geq 0$. It is important for much of what follows to note that when $A_{1}$ is non-singular, the pencil $\sigma_{\gamma[0, \infty)}\left[A_{1}, A_{2}\right]$ is nonsingular if and only if the product $A_{1}^{-1} A_{2}$ has no negative eigenvalues.

The next lemma describes a simple necessary condition, expressed in terms of matrix pencils, for a strong CQLF to exist for two stable LTI systems. This result concerns general $n$-dimensional systems and is well known in the literature. For instance, see [2].

Lemma 2.1 If the stable LTI systems $\Sigma_{A_{1}}, \Sigma_{A_{2}}$ have a strong CQLF, then both of the matrix pencils $\sigma_{\gamma[0, \infty)}\left[A_{1}, A_{2}\right]$ and $\sigma_{\gamma[0, \infty)}\left[A_{1}^{-1}, A_{2}\right]$ are nonsingular. Equivalently, the matrix products $A_{1}^{-1} A_{2}$, $A_{1} A_{2}$ have no negative eigenvalues.

It is important to note that the existence of a strong CQLF for a family of LTI systems is invariant under a change of basis transformation. This is recorded in the following straightforward and well-known lemma, the proof of which involves verifying that if $T \in \mathbb{R}^{n \times n}$ is non-singular, then $A^{T} P+P A<0$ if and only if $\left(T^{-1} A T\right)^{T}\left(T^{T} P T\right)+\left(T^{T} P T\right)\left(T^{-1} A T\right)<0$.

Lemma 2.2 Let $\Sigma_{A_{1}}, \Sigma_{A_{2}}, \ldots, \Sigma_{A_{M}}$ be a family of stable LTI systems and let $T$ be a non-singular matrix in $\mathbb{R}^{n \times n}$. For $i \in\{1, \ldots M\}$, define $\tilde{A}_{i}=T^{-1} A_{i} T$. Then the systems $\Sigma_{A_{1}}, \Sigma_{A_{2}}, \ldots, \Sigma_{A_{M}}$ have a strong CQLF if and only if the systems $\Sigma_{\tilde{A_{1}}}, \Sigma_{\tilde{A_{2}}}, \ldots, \Sigma_{\tilde{A_{M}}}$ have a strong CQLF

The next two lemmas are concerned with pairs of systems whose system matrices differ by rank 1 . First of all we note that for such systems, one of the two pencils in Lemma 2.1 can never be singular. Proofs of Lemmas 2.3 and 2.4 can be found in [5] and [8] respectively.

Lemma 2.3 Let $A, A+B \in \mathbb{R}^{n \times n}$ be Hurwitz with $\operatorname{rank}(B)=1$. Then the matrix product $A^{-1}(A+B)$ has no negative eigenvalues. Equivalently, the matrix pencil $\sigma_{\gamma[0, \infty)}[A, A+B]$ is non-singular.
Lemma 2.4 Let $A, B \in \mathbb{R}^{n \times n}$ with $A$ Hurwitz and $\operatorname{rank}(B)=1$. Suppose that for some $\lambda_{0}>0$, the matrix product $A\left(A+\lambda_{0} B\right)$ has a negative eigenvalue (the pencil $\sigma_{\gamma[0, \infty)}\left[A^{-1}, A+\lambda_{0} B\right]$ is singular). Then for all $\lambda \geq \lambda_{0}$, the product $A(A+\lambda B)$ has a negative eigenvalue (the pencil $\sigma_{\gamma[0, \infty)}\left[A^{-1}, A+\lambda B\right]$ is singular).

The following theorem, first proven in [10], considers pairs of stable LTI systems for which no strong CQLF exists, but for which a weak CQLF exists with $Q_{i}$, $i \in\{1,2\}$, of rank $n-1$ and establishes a set of easily verifiable algebraic conditions, that are satisfied when such a weak CQLF exists. It will be later shown that these conditions are found to play an important role in the question of the existence of strong CQLF's for general LTI systems.

Theorem 2.1 [10] Let $A_{1}, A_{2}$ be two Hurwitz matrices in $\mathbb{R}^{n \times n}$ such that a solution $P=P^{T} \geq 0$ exists to the non-strict Lyapunov Equations

$$
A_{i}^{T} P+P A_{i}=-Q_{i} \leq 0, i \in\{1,2\}
$$

for some positive semi-definite matrices $Q_{1}, Q_{2}$ both of rank $n-1$. Furthermore suppose that no strong CQLF exists for $\Sigma_{A_{1}}$ and $\Sigma_{A_{2}}$. Under these conditions, at least one of the pencils $\sigma_{\gamma[0, \infty)}\left[A_{1}, A_{2}\right]$, $\sigma_{\gamma[0, \infty)}\left[A_{1}, A_{2}^{-1}\right]$ is singular. Equivalently, at least one of the matrix products $A_{1} A_{2}$ and $A_{1} A_{2}^{-1}$ has a real negative eigenvalue.

The main aim of the rest of the paper is to show how this result provides a unifying framework for two well-known quadratic stability criteria.

Corollary 2.1 Let $A_{1}, A_{2}$ be two Hurwitz matrices in $\mathbb{R}^{n \times n}$. A necessary condition for the existence of a strong CQLF is that the matrix products $A_{1} A(k)$ and $A_{1} A(k)^{-1}$ have no real negative eigenvalues for all $k \in[0,1]$ where $A(k)=A_{1}+k\left(A_{2}-A_{1}\right)$.

\section{Some new perspectives on old results}

The CQLF existence problem for a finite number of LTI systems is recognised as an analytical problem of extreme difficulty. Although this problem can be solved efficiently numerically using linear matrix inequalities [1], closed-form necessary and sufficient conditions for the existence of a CQLF are currently only known for a few special cases of system classes; in particular, for the case of pairs of second order LTI systems [11], and for pairs of $n$-dimensional systems whose systems matrices differ by a rank-1 matrix. In this section we show that both of these cases is a special case of Theorem 2.1. Further, this analysis leads to a new formulation of the SISO circle criterion. 


\subsection{Second order systems}

In this section we illustrate the use of Theorem 2.1. We let $\Sigma_{A_{1}}$ and $\Sigma_{A_{2}}$ be stable LTI systems with $A_{1}, A_{2} \in \mathbb{R}^{2 \times 2}$. The following facts follow trivially for second order systems.

(a) If a strong CQLF exists for $\Sigma_{A_{1}}$ and $\Sigma_{A_{2}}$ then the pencils $\sigma_{\gamma[0, \infty)}\left[A_{1}, A_{2}\right]$ and $\sigma_{\gamma[0, \infty)}\left[A_{1}, A_{2}^{-1}\right]$ are necessarily Hurwitz.

(b) If $A_{1}$ and $A_{2}$ satisfy the non-strict Lyapunov equations (3) then the matrices $Q_{1}$ and $Q_{2}$ are both rank 1 (rank $n-1)$.

(c) If a strong CQLF does not exist for $\Sigma_{A_{1}}$ and $\Sigma_{A_{2}}$ then a positive constant $d$ exists such that a strong CQLF exists for $\Sigma_{A_{1}-d I}$ and $\Sigma_{A_{2}}$. By continuity a non-negative $d_{1}<d$ exists such that $A_{1}-d_{1} I$ and $A_{2}$ satisfy Theorem 2.1 and one of the pencils $\sigma_{\gamma[0, \infty)}\left[A_{1}-d_{1} I, A_{2}\right]$ and $\sigma_{\gamma[0, \infty)}\left[A_{1}-\right.$ $\left.d_{1} I, A_{2}^{-1}\right]$ is necessarily singular. Hence, it follows that one of the pencils $\sigma_{\gamma[0, \infty)}\left[A_{1}, A_{2}\right]$ and $\sigma_{\gamma[0, \infty)}\left[A_{1}, A_{2}^{-1}\right]$ is not Hurwitz.

Items (a)-(c) establish the following facts. Given two stable second order LTI systems $\Sigma_{A_{1}}$ and $\Sigma_{A_{2}}$, a necessary condition for the existence of a strong CQLF is that the pencils $\sigma_{\gamma[0, \infty)}\left[A_{1}, A_{2}\right]$ and $\sigma_{\gamma[0, \infty)}\left[A_{1}, A_{2}^{-1}\right]$ are Hurwitz. Conversely, a necessary condition for the non-existence of a strong CQLF is that one of the pencils $\sigma_{\gamma[0, \infty)}\left[A_{1}, A_{2}\right]$ and $\sigma_{\gamma[0, \infty)}\left[A_{1}, A_{2}^{-1}\right]$ is not Hurwitz. Together with Theorem 2.1 these conditions yield the following known result $[11,2]$ :

A necessary and sufficient condition for the LTI systems $\Sigma_{A_{1}}$ and $\Sigma_{A_{2}}, A_{1}, A_{2} \in \mathbb{R}^{2 \times 2}$, to have a strong CQLF is that the pencils $\sigma_{\gamma[0, \infty)}\left[A_{1}, A_{2}\right]$ and $\sigma_{\gamma[0, \infty)}\left[A_{1}, A_{2}^{-1}\right]$ are Hurwitz.

\subsection{General systems: The SISO Circle Criterion}

The result presented in Theorem 3.1 below is concerned with the problem of determining necessary and sufficient conditions for a strong CQLF to exist for two LTI systems $\Sigma_{A}, \Sigma_{A-g k^{T}}$ with $A$ and $A-g k^{T}$ in companion form. The result of this theorem can be thought of as a time-domain formulation of the circle criterion. Our main goal in this section is to indicate how Theorem 2.1 above can provide a general setting in which to approach the problem of strong CQLF existence for pairs of LTI systems. With this in mind, the proof we present below illustrates the general nature of the conditions described in Theorem 2.1 by demonstrating that known results for second order systems and those systems covered by the circle criterion can be treated within the framework of the Theorem.

Before stating Theorem 3.1 we make the following preliminary comments.

\section{Preliminaries to Theorem 3.1:}

(i) Because both $A$ and $A-g k^{T}$ are in companion form, we may write;

$$
\begin{gathered}
A=\left(\begin{array}{llllr}
0 & 1 & 0 & \ldots & 0 \\
0 & 0 & 1 & \ldots & 0 \\
\vdots & & & & \\
0 & 0 & 0 & \ldots & 1 \\
-a_{0} & -a_{1} & -a_{2} & \ldots & -a_{n-1}
\end{array}\right), \\
g=\left(\begin{array}{c}
0 \\
0 \\
\vdots \\
0 \\
1
\end{array}\right), k=\left(\begin{array}{c}
k_{0} \\
k_{1} \\
\vdots \\
k_{n-2} \\
k_{n-1}
\end{array}\right) .
\end{gathered}
$$

(ii) If we define the rational function $\Gamma(\omega)$ by

$$
\Gamma(\omega)=1+\operatorname{Re}\left\{k^{T}(j \omega I-A)^{-1} g\right\}
$$

then it follows from the circle criterion that there is a strong CQLF for $\Sigma_{A}, \Sigma_{A-g k^{T}}$ if and only if $\Gamma(\omega)>0$ for all real $\omega([4,7,12])$. Furthermore, if we define $\lambda_{c}$ to be the supremum of those $\lambda>0$ such that $\Sigma_{A}, \Sigma_{A-\lambda g k^{T}}$ have a strong CQLF, then (provided $\lambda_{c}<\infty$ ), for $\Gamma_{c}(\omega)=1+\operatorname{Re}\left\{\lambda_{c} k^{T}(j \omega I-A)^{-1} g\right\}$ we have that $\Gamma_{c}(\omega) \geq 0$ for all real $\omega$ and $\Gamma_{c}\left(\omega_{0}\right)=0$ for some $\omega_{0}$.

(iii) We shall need to know how the coefficients of the numerator of $\Gamma$ are related to the entries of $A$ and $k$. As pointed out by Kalman in [4], for any vector $k$ in $\mathbb{R}^{n}$;

$k^{T}(s I-A)^{-1} g=\frac{k_{0}+k_{1} s+\ldots+k_{n-1} s^{n-1}}{\operatorname{det}(s I-A)}$

and from this it follows that we can write

$$
\Gamma(\omega)=\frac{p_{1}(\omega)}{\operatorname{det}\left(\omega^{2} I+A^{2}\right)}
$$

where $p_{1}$ is a polynomial in $\omega$ of degree $2 n$. Furthermore, as $\operatorname{det}(s I-A)=a_{0}+a_{1} s+\ldots+$ $a_{n-1} s^{n-1}+s^{n}$, we can write

$$
\begin{aligned}
p_{1}(\omega) & =\left[\operatorname{det}\left(\omega^{2} I+A^{2}\right)\right] \\
& +\left[k_{0} a_{0}+\left(-k_{0} a_{2}+k_{1} a_{1}-k_{2} a_{0}\right) \omega^{2}\right. \\
& +\left(k_{0} a_{4}-k_{1} a_{3}+k_{2} a_{2}-k_{3} a_{1}+k_{4} a_{0}\right) \omega^{4} \\
+\ldots & \left.+\left(-k_{n-2}+k_{n-1} a_{n-1}\right) w^{2 n-2}\right] .
\end{aligned}
$$


Note that only even powers of $\omega$ appear in $p_{1}(\omega)$ so that we can also consider $p_{1}$ to be a polynomial in $\omega^{2}$. Now, it follows from (6) that for a given $A \in \mathbb{R}^{n \times n}$ in companion form, the relationship between the entries of the vector $k$ and the coefficients of $p_{1}$ (considered as a polynomial in $\omega^{2}$ ) is described by the affine mapping (from $\mathbb{R}^{n}$ to $\mathbb{R}^{n}$ )

$$
T(k)=\Theta(A)+L(A) k
$$

where $\Theta$ is a vector that depends on the entries of $A$ and $L(A)$ is the linear map given by the matrix (in $\mathbb{R}^{n \times n}$ )

$$
\left(\begin{array}{cccccc}
a_{0} & 0 & 0 & \ldots & 0 & 0 \\
-a_{2} & a_{1} & -a_{0} & \ldots & 0 & 0 \\
\vdots & \vdots & & \vdots & \vdots & \\
0 & 0 & 0 & \ldots & -1 & a_{n-1}
\end{array}\right)
$$

(iv) Now note that the determinant of $L(A)$ is not independent of the entries of $A$, and hence is not uniformly zero (for instance, the product term $a_{0} a_{1} a_{2} \ldots a_{n-1}$ can only appear once in the expression for the determinant). Thus, for any companion matrix $A$ such that $L(A)$ is singular, it is possible to find another matrix $A^{\prime}$, also in companion form, arbitrarily close to $A$ with $L\left(A^{\prime}\right)$ invertible by perturbing the entries $a_{0}, a_{1}, \ldots, a_{n-1}$

Theorem 3.1 Let $A, A-g k^{T}$ be two Hurwitz matrices in companion form in $\mathbb{R}^{n \times n}$ where $g, k$ are column vectors in $\mathbb{R}^{n}$. Assume that the matrix $L(A)$ defined by (8) is non-singular. Then a necessary and sufficient condition for a strong CQLF to exist for the systems $\Sigma_{A}, \Sigma_{A-g k^{T}}$ is that the matrix product $A\left(A-g k^{T}\right)$ has no negative eigenvalues or equivalently, that the matrix pencil $\sigma_{\gamma[0, \infty)}\left[A^{-1}, A-g k^{T}\right]$ is non-singular.

\section{Comments on Theorem 3.1:}

(i) It is important to point out that the assumption that $L(A)$ is invertible is not a very strong restriction on $A$. In fact if we identify the companion matrix

$$
A=\left(\begin{array}{llllr}
0 & 1 & 0 & \ldots & 0 \\
0 & 0 & 1 & \ldots & 0 \\
\vdots & & & & \\
0 & 0 & 0 & \ldots & 1 \\
-a_{0} & -a_{1} & -a_{2} & \ldots & -a_{n-1}
\end{array}\right)
$$

with the vector $\left(a_{0}, a_{1}, \ldots, a_{n-1}\right)^{T}$ in $\mathbb{R}^{n}$, then the set of those companion matrices for which $L(A)$ is singular would have Lebesgue measure zero. (ii) Moreover from a practical point of view, if you have two systems in companion form, it is a simple matter to check if $L(A)$ is invertible or not, and if not to adjust the parameters of $A$ to make $L(A)$ invertible.

(iii) Theorem 3.1 can also be extended to the case where the matrices $A$ and $A-g k^{T}$ are not assumed to be in companion form by following Meyer's proof of the extended Kalman Yakubovich Popov lemma given in [6] - corresponding to the general case of systems differing by a rank one perturbation.

The crucial point in the proof of Theorem 3.1 is provided by the following lemma which also indicates the relevance of Theorem 2.1 in this context. In the lemma we consider the situation where two systems have just ceased to have a CQLF and we show that under these circumstances there are two systems arbitrarily close to the original systems that satisfy the conditions of Theorem 2.1.

Lemma 3.1 Let $A, A-g k^{T} \in \mathbb{R}^{n \times n}$ be Hurwitz matrices in companion form with $L(A)$ invertible. Suppose that there is no strong CQLF for $\Sigma_{A}$ and $\Sigma_{A-g k^{T}}$. Furthermore suppose that there is a strong CQLF for $\Sigma_{A}$ and $\Sigma_{A-\lambda g k^{T}}$ for all $\lambda$ with $0<\lambda<1$. Then given any $\epsilon>0$, there is some $k^{\prime} \in \mathbb{R}^{n}$ with $\left\|k-k^{\prime}\right\|<\epsilon$ for which there exists a matrix $P=P^{T} \geq 0$ satisfying

$$
\begin{aligned}
A^{T} P+P A & =Q_{1} \leq 0 \\
\left(A-g k^{\prime T}\right)^{T} P+P\left(A-g k^{\prime T}\right) & =Q_{2} \leq 0
\end{aligned}
$$

with $\operatorname{rank}\left(Q_{1}\right)=n-1, \operatorname{rank}\left(Q_{2}\right)=n-1$.

The proof of Lemma 3.1 is quite long and involved. For details, consult the technical report [8].

Comment: It follows from Theorem 2.1 and Lemma 2.3 that each of the matrix products $A\left(A-g k^{\prime T}\right)$ occurring in the above lemma has a negative real eigenvalue. This in turn implies by the continuous dependence of the eigenvalues of a matrix upon its entries that the matrix product $A\left(A-g k^{T}\right)$ has a negative real eigenvalue.

\section{Proof of Theorem 3.1:}

If there is a strong CQLF for the systems $\Sigma_{A}, \Sigma_{A-g k^{T}}$ given by $V(x)=x^{T} P x$, then it follows from Lemma 2.1 that the product $A\left(A-g k^{T}\right)$ has no negative eigenvalue.

Conversely, suppose there is no strong CQLF for $\Sigma_{A}, \Sigma_{A-g k^{T}}$. Then it follows from the continuous dependence of the eigenvalues of a matrix on 
the entries of the matrix that for small enough values of $\lambda>0$, the systems $\Sigma_{A}, \Sigma_{A-\lambda g k^{T}}$ will have a strong CQLF. Define $\lambda_{c}=\sup \{\lambda>0$ : $\Sigma_{A}$ and $\Sigma_{A-\lambda g k^{T}}$ have a strong CQLF $\}$. Then $\lambda_{c} \leq$ 1 and $\Sigma_{A}$ and $\Sigma_{A-\lambda_{c} g k^{T}}$ satisfy the conditions of Lemma 3.1. Thus it follows from the comment above that the matrix product $A\left(A-\lambda_{c} g k^{T}\right)$ has a negative real eigenvalue. It now follows immediately from Lemma 2.4 that the matrix product $A\left(A-g k^{T}\right)$ has a negative real eigenvalue. Q.E.D

\section{General Case and the KYP Lemma in the time domain}

In view of the preceding results and their connection with the SISO circle criterion, it is natural to ask whether or not the generalized Kalman-YakubovichPopov (KYP) lemma due to Meyer [6] admits a similar time-domain formulation. We provide the answer to this question in this section.

Meyer's result established that for two stable LTI systems $\Sigma_{A}, \Sigma_{A-b c^{T}} A \in \mathbb{R}^{n \times n}, b, c \in \mathbb{R}^{n}$, a sufficient condition for the existence of a CQLF is given by

$1+\operatorname{Re}\left\{c^{T}(j \omega I-A)^{-1} b\right\}>0$ for all $\omega \in \mathbb{R}$.

Note that no assumption about $A$ and $A-b c^{T}$ being in companion form is made here (hence the change in notation to avoid confusion). We shall now show that the condition (9) is also necessary for the existence of a CQLF; in fact the matrix product condition described in Theorem 3.1 is equivalent to (9). This extends the work presented in [9] where an equivalent time-domain formulation of the SISO circle criterion was given.

The following standard lemma is needed for the proof of Theorem 4.1. For details consult [3].

Lemma 4.1 Let $A \in \mathbb{R}^{n \times n}, b, c \in \mathbb{R}^{n}$. Then for any complex $s, \operatorname{det}\left(c^{T}(s I-A)^{-1} b\right)$ is equal to the expression

$$
\frac{\operatorname{det}\left(s I-\left(A-b c^{T}\right)\right)-\operatorname{det}(s I-A)}{\operatorname{det}(s I-A)} .
$$

Theorem 4.1 Let $A \in \mathbb{R}^{n \times n}, b, c \in \mathbb{R}^{n}$ be such that $A$ and $A-b c^{T}$ are Hurwitz matrices. Suppose that $A\left(A-b c^{T}\right)$ has no negative eigenvalues. Then the condition (9) holds.

Proof: Suppose that $A\left(A-b c^{T}\right)$ has no negative eigenvalues. Then as $A$ and $A-b c^{T}$ are both Hurwitz their determinants will have the same sign, so it follows that for all $\lambda>0$

$\operatorname{det}\left(\lambda I-\left(A-b c^{T}\right) A\right)=\operatorname{det}\left(\lambda I-A^{2}-b c^{T} A\right)>0$
Without loss of generality, we may assume that $b c^{T}$ is in one the Jordan canonical forms

$$
\begin{aligned}
& \text { (i) } B=\left(\begin{array}{cccc}
c & 0 & \ldots & 0 \\
0 & \ldots & \ldots & 0 \\
\vdots & & & \\
0 & \ldots & \ldots & 0
\end{array}\right) \text {, } \\
& \text { (ii) } B=\left(\begin{array}{cccc}
0 & \ldots & \ldots & 0 \\
1 & \ldots & \ldots & 0 \\
\vdots & & & \\
0 & \ldots & \ldots & 0
\end{array}\right) \text {. }
\end{aligned}
$$

If $b c^{T}$ is in either of the above forms then it follows that the expressions

$$
\operatorname{det}\left(\lambda I-A^{2}-b c^{T} A\right)
$$

and

$$
\operatorname{Re}\left\{\operatorname{det}\left(\lambda I-A^{2}-b c^{T} A-\sqrt{\lambda} j b c^{T}\right)\right\},
$$

are identical. Thus, writing $\lambda=\omega^{2}$ we have that for all real $\omega$

$$
\operatorname{Re}\left\{\operatorname{det}\left(\omega^{2} I-A^{2}-b c^{T} A-j \omega b c^{T}\right)\right\}>0 .
$$

It now follows, after a short calculation ([8]) that for all $\omega \in \mathbb{R}$

$1+\operatorname{Re}\left\{\frac{\operatorname{det}\left(j \omega I-\left(A-b c^{T}\right)\right)-\operatorname{det}(j \omega I-A)}{\operatorname{det}(j \omega I-A)}\right\}>0$

and hence from Lemma 4.1 that for all real $\omega$

$$
1+\operatorname{Re}\left\{c^{T}(j \omega I-A)^{-1} b\right\}>0
$$

as claimed. Q.E.D.

\section{Comments on Theorem 4.1}

(i) The above result establishes that condition (9) is necessary as well as sufficient for the existence of a CQLF for $\Sigma_{A}, \Sigma_{A-b c^{T}}$. To the best of the authors' knowledge, this is a new result. To see this, suppose $\Sigma_{A}, \Sigma_{A-b c^{T}}$ have a strong CQLF. Then it follows from Lemma 2.1 that the product $A\left(A-b c^{T}\right)$ has no negative eigenvalues and hence by Theorem 4.1 condition (9) must hold. Note that this also establishes that $A\left(A-b c^{T}\right)$ having no negative eigenvalues is an equivalent time-domain formulation of the condition (9).

(ii) If the matrix product $A\left(A-b c^{T}\right)$ has no negative eigenvalues then from the above theorem and the original result of Meyer [6], it follows that $\Sigma_{A}$ and $\Sigma_{A-b c^{T}}$ have a strong CQLF. This together with Lemma 2.1 gives the following necessary and sufficient condition for a CQLF to exist for two stable LTI systems differing by rank one. 
Theorem 4.2 Let $A, B \quad \in \quad \mathbb{R}^{n \times n}$ with $\operatorname{rank}(B)=1$ and $A, A+B$ Hurwitz. Then there is a strong CQLF for the stable LTI systems $\Sigma_{A}$ and $\Sigma_{A+B}$ if and only if the matrix product $A(A+B)$ has no negative eigenvalues.

\section{Conclusions}

In this paper, we have presented a result on common quadratic Lyapunov functions, namely Theorem 2.1, and demonstrated that a number of well-known CQLF existence criteria fall within the framework of this result. Considerable empirical evidence indicates to the authors that other system classes will admit treatment within this same framework, and that necessary and sufficient conditions for CQLF existence for these classes may be obtained using Theorem 2.1. The determination of such system classes is currently the subject of ongoing research by the authors and any results obtained in this direction will be reported in future publications.

\section{Acknowledgements}

This work was partially supported by the European Union funded research training network Multi-Agent Control, HPRN-CT-1999-00107 ${ }^{1}$ and by the Enterprise Ireland grant SC/2000/084/Y. Neither the European Union or Enterprise Ireland is responsible for any use of data appearing in this publication.

\section{References}

[1] S. Boyd and Q. Yang. Structured and simultaneous Lyapunov functions for system stability problems. Internat. J. Control, 49(6):22152240, 1989.

[2] N. Cohen and N. Lewkowicz. Convex invertible cones and the Lyapunov equation. Lin. Alg. and its Appl., 250(1):105-131, 1997.

[3] T Kailath. Linear Systems. Prentice Hall, New Jersey, 1980.

[4] R.E. Kalman. Lyapunov functions for the problem of Lur'e in automatic control. Proc. Natl. Acad. Sci., 49(2):201-205, 1963.

[5] T. Laffey, R. Shorten, and F. O'Cairbre. On the stability of convex sums of rank-1 perturbed matrices. In Proceedings of the American Control Conference, 2002.

[6] K. R. Meyer. On the existence of Lyapunov functions for the problem of Lur'e. SIAM Journal of Control, 3(3):373-383, 1966.

\footnotetext{
${ }^{1}$ This work is the sole responsibility of the authors and does not reflect the European Union's opinion
}

[7] K. Narendra and R. Goldwyn. A geometrical criterion for the stability of certain non-linear nonautonomous systems. IEEE Transactions on Circuit Theory, 11(3):406-407, 1964.

[8] R. Shorten, O. Mason, F. O' Cairbre, and P. Curran. A unifying framework for the circle criterion and other quadratic stability criteria. Technical Report NUIM/SS/2003/03, NUI Maynooth, 2003.

[9] R. N. Shorten and K. S. Narendra. On common quadratic Lyapunov functions for pairs of stable lti systems whose system matrices are in companion form. IEEE Transactions on Automatic Control, 48(4):618-621, 2003.

[10] R. N. Shorten, K. S. Narendra, and O. Mason. A result on common quadratic Lyapunov functions. IEEE Transactions on Automatic Control, 48(1):110-113, 2003.

[11] Robert N. Shorten and Kumpati Narendra. Necessary and sufficient conditions for the existence of a common quadratic Lyapunov function for a finite number of stable second order linear time-invariant systems. International Journal of Adaptive Control and Signal Processing, 16:709-728, 2002.

[12] J. Willems. The circle criterion and quadratic Lyapunov functions for stability analysis. IEEE Transactions on Automatic Control, 18:184, 1973. 